\title{
An Approach to Knowledge Acquisition for the Hull Form Design of Fishing Crafts
}

\author{
- First Report : Generic Database for Hull Form Representation-
}

\author{
by Takeo Koyama*, member Hiroyuki Yamato*, member \\ Glenn D. Aguilar**, member
}

\begin{abstract}
Summary
A knowledge based design system for fishing vessels consisting of a consultation component, a generic database/representation program and statistics based tools for analysis was developed. An overview of the system is discussed in this paper with the database system and procedure described in detail. The database generation system consisted of an on-screen digitizing program requiring scanned images of two dimensional lines. The digitized hull was stored in a database as an instance consisting of a class defined in an object oriented system. Upright hydrostatic calculation were also included and used for checking the scaling error of the system. The program was developed to build up knowledge about existing hull forms and when the database is of sufficient size, statistical tools and techniques as well as other useful, relevant tools will be applied to derive knowledge on hull design.
\end{abstract}

\section{Introduction}

The primary objective of this research is to develop a knowledge based system for the design of fishing crafts. Design practice is well established in this field particularly in developed countries with a significant fishing sector. The kowledge and expertise available from such a country could be used by others which need them most, particulary the developing countries. Multiple factors have significant influence in the introduction of such but the major intention of this work is to at least provide a capability for modern fishing boat design practice to be available in a form integrating engineering design practice with knowledge drawn from design experience.

A variety of data and references exist for the design of fishing vessels. Most are in the form of plans, reports on performance including resistance, stabiliy, seakeeping characteristics and other such information. Fishing craft are also among the most varied type of structure designed by man. Craft designs, particularly smaller types, are highly localized both in form and function. A design system has to take into consideration such characteristics if a developed design is to be readily accepted by local fishermen.

A knowledge based design system must contain a

\footnotetext{
* Faculty of Engineering, University of Tokyo

** Graduate School, Faculty of Engineering, University of Tokyo.
}

Received 8th Jan 1993

Read at the Spring meeting 19, 20th May 1993 powerful knowledge base consisting of established design practice rules and a wide variety of hull forms, appropriate inference engine, and user friendly interface integrated into the design spiral modules.

Writing procedural or high level language programs to solve problems and configuring existing software for specific purposes consist most of the activities in the development of any system. However, recent advances in computing technology has introduced several paradigms which have quite a tremendous impact on the evolution and process of creating problem solving systems.

Of obvious interest and practical utility to ship design systems is the object oriented design and analysis point of view coupled with the practical application of expert systems.

Several notable sysems have evolved in the definition and employment of a product model which is increasingly gaining widespread acceptance as the framework for CIM (Computer Integrated Manufacturing) systems. A lot of work has also been conducted in the inclusion of expertise into the design systems resulting in a class of knowledge based applications.

As a prelude to the implementation of an object oriented system, the Object Modelling Technique ( OMT ) developed by Rumblaugh and others ${ }^{1)}$ has proven to be quite useful and was employed in representing classes and relationships between classes as well as the functional description of the systems.

\section{Description of System components}

The knowledge based design system is designed as a multiple-object system (using the OMT description) as 
described in Fig. 1. The present focus is on the development of a knowledge acquistion system which seeks to capture expertise in the form of expert knowledge from both experts and published data and represent such knowledge for use by the design system.

Three components of the knowledge acquisition system are being utilized for the construction of a knowledge base. The first is a consultation system for the classification of elements based on attributes describing the element. The intention is to construct decision making rules for choosing between elements. A second component is used for the digitization and representation of hull forms from published sources. The third component utilizes new approaches in knowledge discovery and statistics to analyze historical data. (Fig. 2)

Knowledge acquisition is acknowledged as one of the most difficult problems in building knowledge based systems ${ }^{2}$. One major difficultly is the wide gap between what the expert or knowledge source knows and the final representation in the computer. The development of an interactive decision making tool making use of grid construct technology, entailments and certainty factor analysis patterned after the kowledge elicitation tools like ETS, Aquinas and Nextra has been started for purposes of decision making ${ }^{3), 4}$.

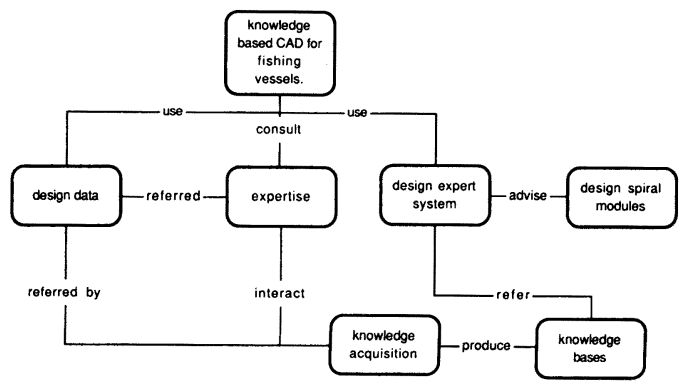

Fig. 1. General system objects.

\section{Geometric Database Construction}

Although classifications and rule representations offer the capability of providing advice for decision, it is inadequate for design purposes ${ }^{5)}$ or other tasks which are categorized as synthesis problems. These tasks require not only classifications, but a combination of algorithmic and highly empirical procedures.

Current design technology is focused on the definition of the product model where the processes describing form (topology, geometry), behavior and function are applied to produce a final design. Many excellent works have been accomplished using such an approach ${ }^{6}$. As there was an obvious need of hull form data on which to base decisions and derive quantitative informations, a program was developed to represenst and store the geometry of hull forms from 2 dimensional lines drawings.

Digitizing is normally done with the use of a digitizer where the position of a pointer and user actions such as clicking a button determines the relative position of a point within a coordinate system. Scanned images has some advantages over digitizers although there also some offsetting disadvantages.

The most important advantage of the scanner is the capability to input colored images which has the poten. tial of adding visualization capabilities to intelligent databases which involve the use of pictures for visualization, advising, tutorial functions and other information related purposes (a picture is worth a thousand words).

The general objects repesenting the entire system is shown in Fig. 3. Objects representing the components of the program itself is shown in Fig. 4. Classes making up the system as implemented in Prograph, a high level visual, data flow and object oriented system. is shown in Fig. 5. Process flow is described in Fig. 6.

In order to preserve accuracy, several precautions have to be taken during the image scanning process:

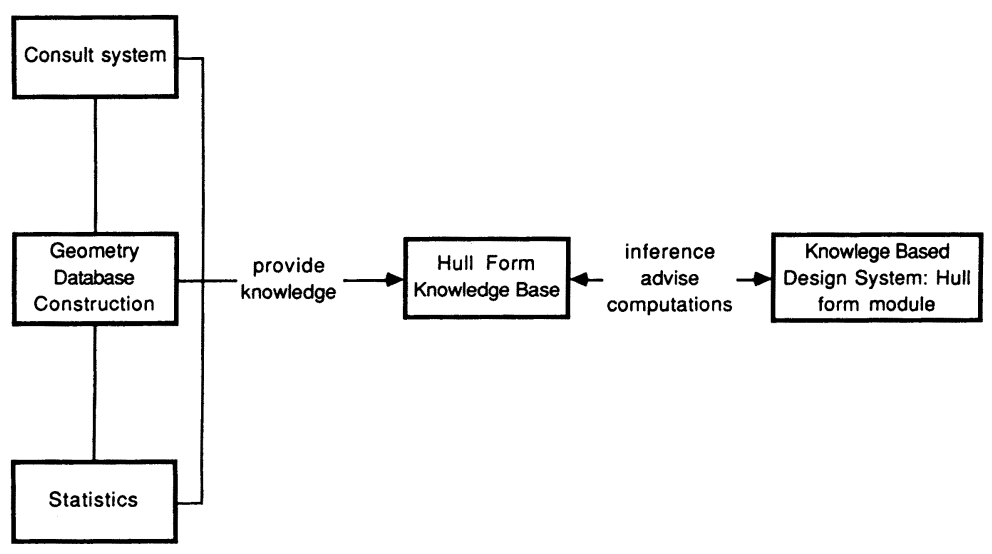

Fig. 2. Knowledge acquisition system objects. 


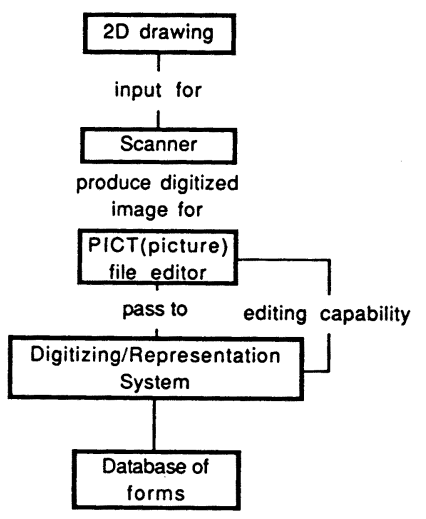

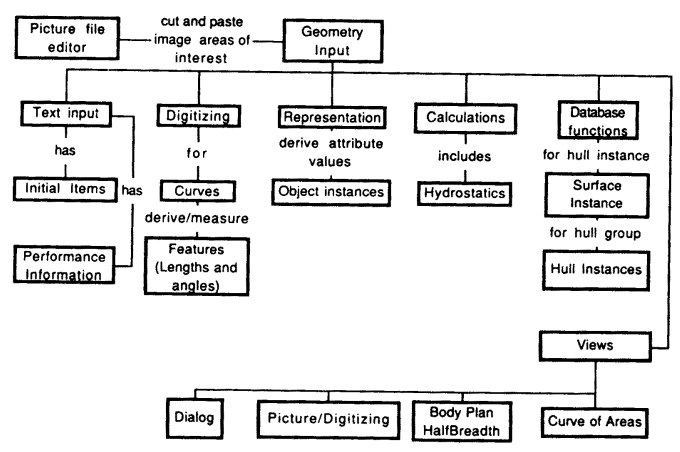

Fig. 4. Object description of geometry input system.

Fig. 3. Objects describing system components.

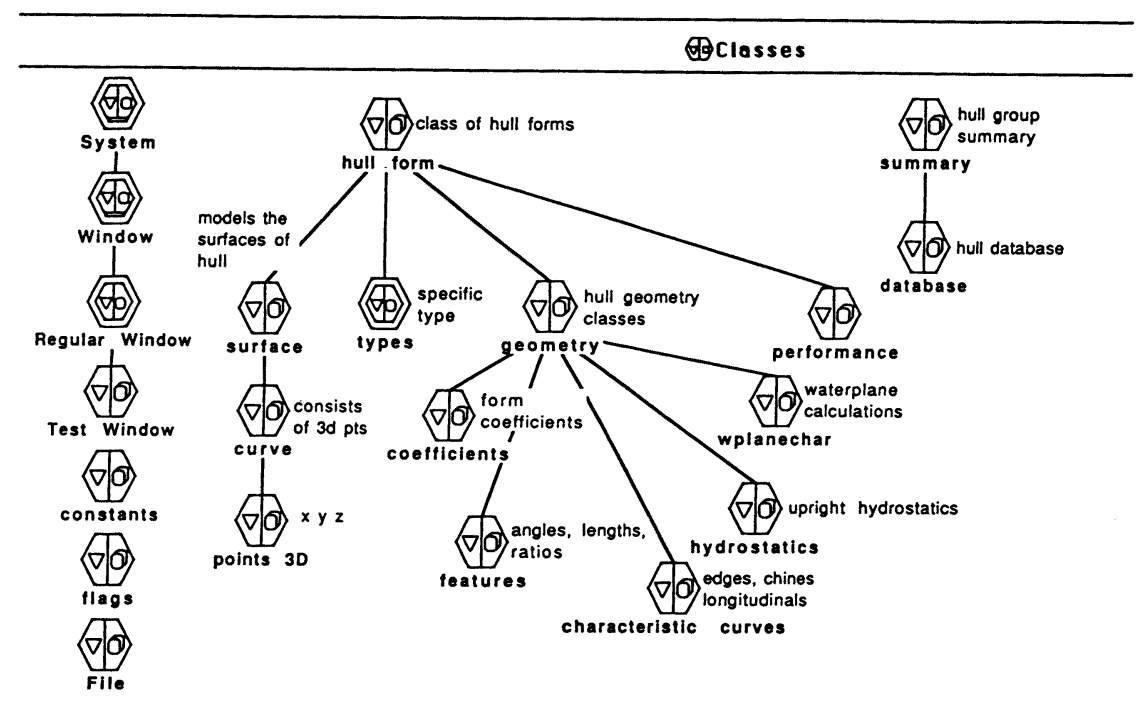

Fig. 5. Implementation objects in Prograph.

1. Take care in aligning the picture with the sides of the scanner. Straight vertical lines are readily produced but when there is a slight slant or the lines at an angle, jagged edges appear.

2. As much as possible, the image should not be scaled by the scanner, this reduces the possibility of built-in error.

3. If the original drawing is too big to fit the digitizing screen, reducing the image using a photocopier was found to be result in more accurate dimensions.

4. Scaling using higher resolutions was also found to reproduce better looking and more pleasing digitizing pictures. Using only black and white during scanning resulted in some loss of some parts of the image.

Once a hull form is scanned, it is then input to a drawing package where the scale is copied and pasted to each drawing. Depending on the drawing package, several possibilities exist for manipulating the hull form. A series of hulls with varied B/D can be created by simply changing the aspect ratio of the picture.

Using the digitizing system initially requires the input of some initial data for scaling and initializing purposes. (Fig. 7) Since the lengths are included with each data image, each section of the lines drawing is scaled before digitizing.

Digitizing requires clicking on the curve points of the image. Getting the values of a point on the curve is accomplished by a two stage process involving the digitized pixels. The cursor is first placed on the point to be digitized and the mouse is clicked. A three pixel by three pixel rectangle centered at the point of the mouse click is searched for a pixel point which is found on the curve (black pixel). Priority weights are assigned to multiple pixel locations, inner pixels (pixels nearest the centerline) get higher priority than those outside. If the whole square is filled with black pixels, the point derived is the center of the rectangle. This was 
found to be more accurate than simply clicking on a perceived point on a curve.

Each curve of interest is then digitized with some descriptions given to describe the curve and its location on the hull. As most drawing files were too big to fit the screen, the images are grouped into three and digitized in turn : the profile aft, body plan and fore aft. Since all the curves are represented by all three, it was no longer necessary to digitize the plan views. First, the aft curves are digitized (Fig. 8), then the stations (Fig. 9 and 10) and finally the fore curves (Fig. 11). Digitization is conducted in the aft to fore direction and from the keel to the sheer line. Each click point is represented by a square on the image. Various buttons, click boxes, display fields and input fields are used during the proc-

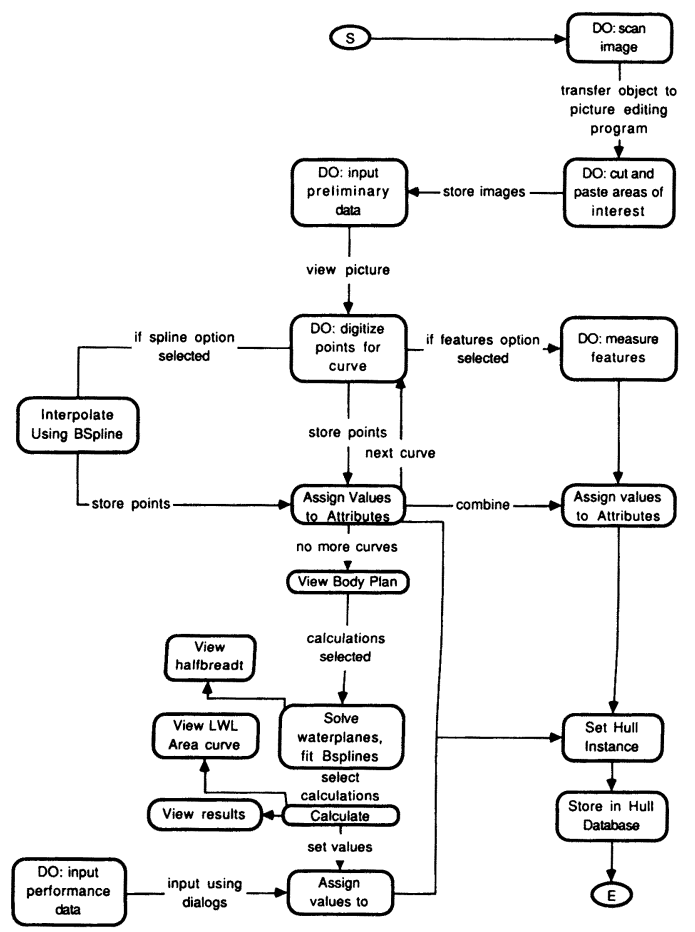

Fig. 6. State diagram for geometry input.

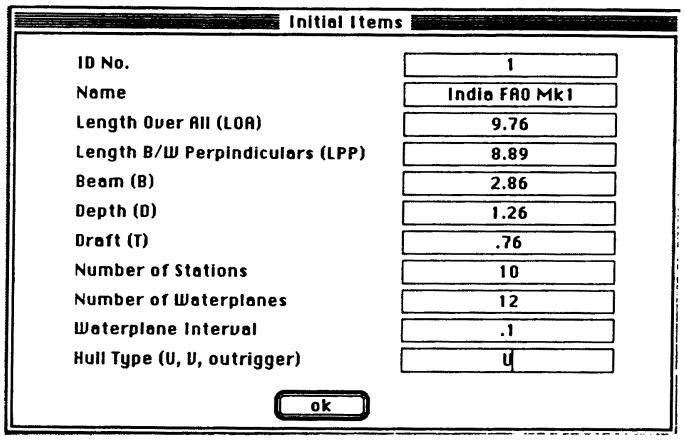

Fig. 7. Initial input items.

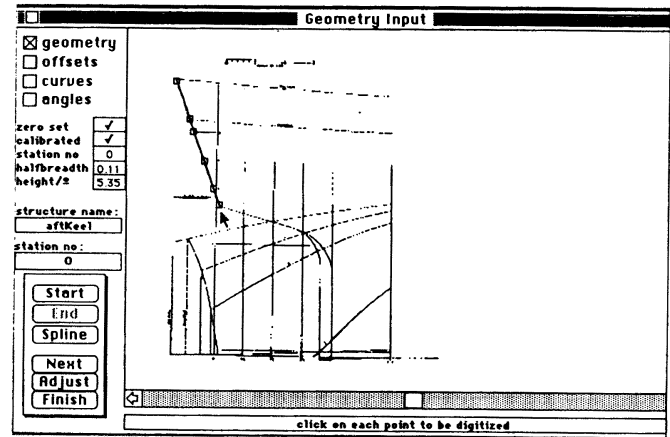

Fig. 8. Aft curves.

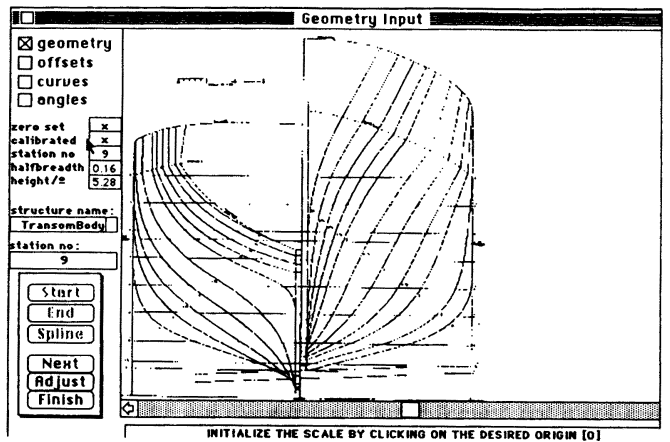

Fig. 9. Stations prior to digitizing.

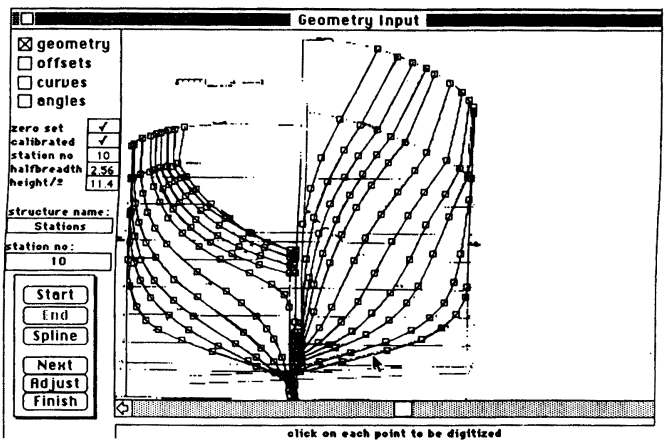

Fig. 10. Stations after digitizing.

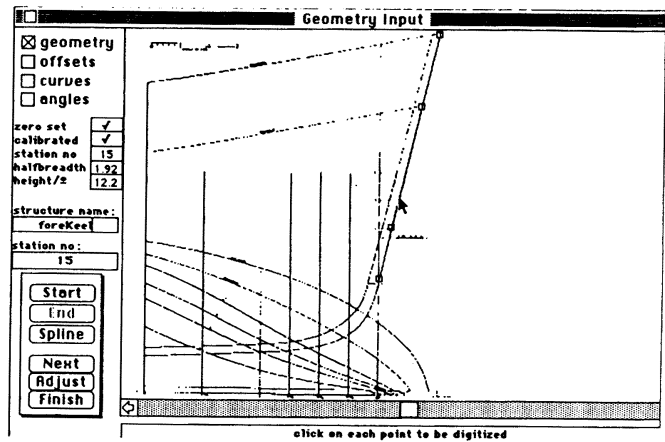

Fig. 11. Fore curves. 
ess. Depending on the complexity of the hull, the process takes up to 10 minutes including scanning the pictured until the hull form is stored in the database.

The development system, Prograph, has data flow computing structures, providing some convenience for simultaneous calculations during the digitization process. While digitizing, waterplane intersections can be calculated. Other options include fitting B-splines to the sections after each curve has been digitized. An interpolating B-spline as developed by Yamaguchi ${ }^{7)}$ defines each station. From each curve, waterplanes are then derived and further calculations conducted. However, as seen from Fig. 12, the body plan as represented by straight line segments connecting the digitzed points provided quite an accurate image of the input picture image, fitting B-splines to the sections after digitizing resulted in noticeable delays.

After digitization, calculations can be conducted on the stations. The first calculation procedure is to find the intersections of the waterlines with the given input curves. An interpolating B-spline curve is then fitted through these points to serve as a smooth and quite fair representation (Fig. 13). The advantages of the B-spline curve for ship hull representation has been espoused by many authors and most commercial as well as proprietary design software employ this curve for geometric modelling. At this level however, the advantages of the B-spline as a representation are not too effective but it is included just the same for future use.

Sections are then recalculated from the waterlines to give intermediate stations. Other calculations are then conducted which include displacement, waterplane characteristics, coefficients and other form parameters. An option to view the curve of areas is included (Fig. 14) to describe the prismatic coefficient and LCB. An option to produce offsets for Macsurf is also included and the resulting plot is shown in Fig. 15.

Another example is shown in Fig. 16 to 18 which represents a chined hull and the resulting curve of areas.

To evaluate the accuracy of the system, four forms were scanned from $\mathrm{FAO}^{8), 9)}$. and digitized to get the

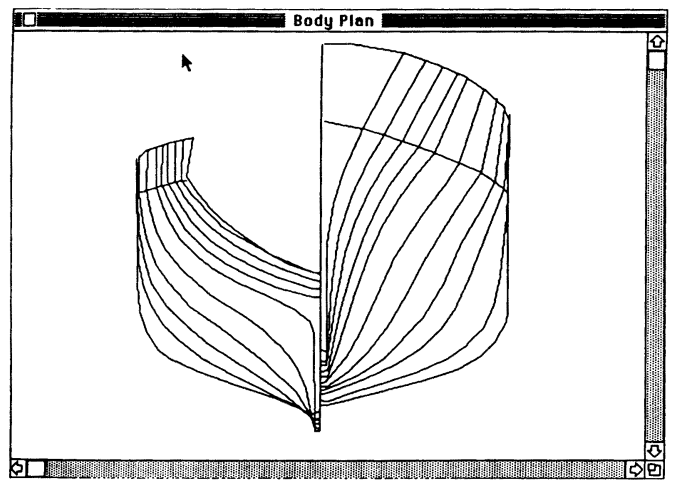

Fig. 12. Plotted body plan from the input points.

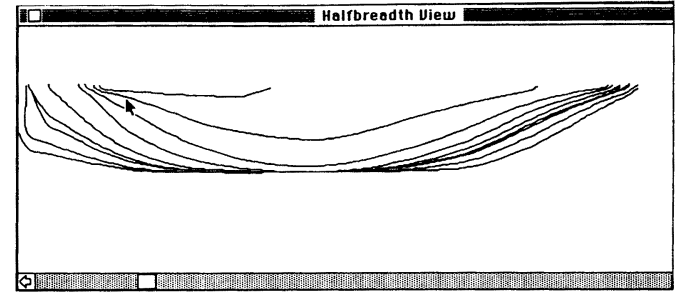

Fig. 13. Waterlines fitted with interpolating B-splines (Yamaguchi 1978).

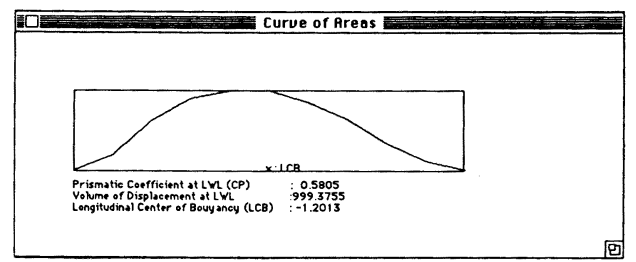

Fig. 14 Plot of the curve of areas at the LWL for the hull.

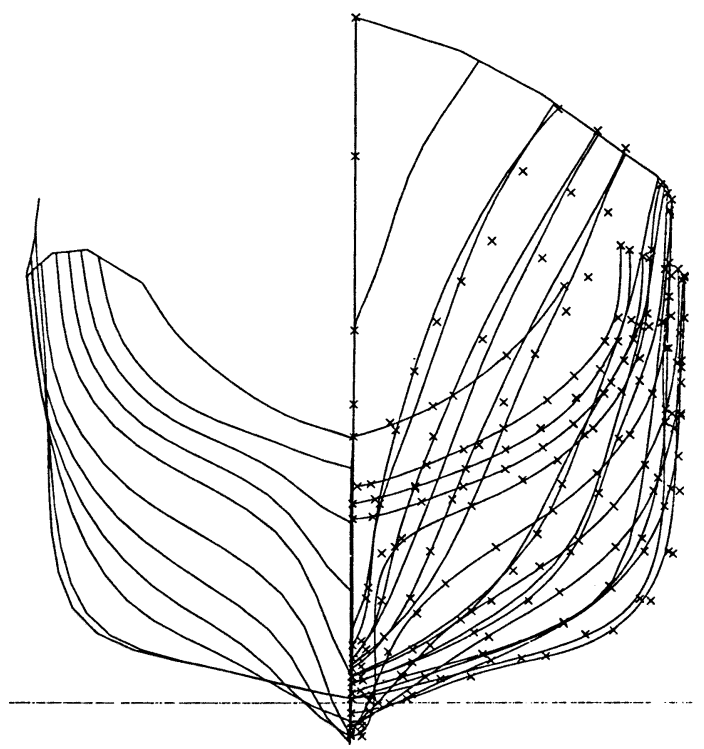

Fig. 15. Macsurf body plot from offsets produced by program.

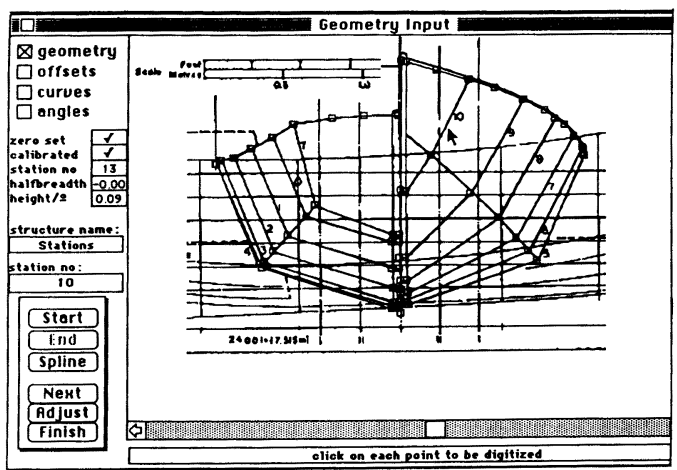

Fig. 16. Digitized chined hull. 
displacement and hydrostatic characteristics and compare with the values given in the reference. The absolute value of the average \% error had a maximum of $16 \%$ for the displacement, CM and CP (Fig. 19). The highest error consistently occurred with an increase in vessel length. Obviously, the reduction in scale contributed quite significantly to the limits of accuracy. Further scanning of a large number of forms should produce an accurate scale factor to be used for different drawing scale values. Further work is continuing in this regard.

The hull form as digitized is then stored in a database. The stored item is an instance of class surface with the attributes set to the digitized values and an instance of performance as solved or given. Hull form groups can be stored in a file with each hull represented by a summary of input data, LWL values and performance data. It is then a matter of using statistical analysis to derive rules or other forms of knowledge from the database.

\section{Statistical Analysis}

Many major advances in statistical techniques have arisen in conjunction with the growth in number and magnitude of databases. A lot of information is available and much knowledge is stored in databases; it is only necessary to find the proper tools and representations to derive appropriate knowledge. Knowledge discovery is the term coined to encompass such an extraction of useful information from data ${ }^{10)}$. Several levels of analysis can be conducted to derive useful rules; starting from the raw data itself, to single vari-

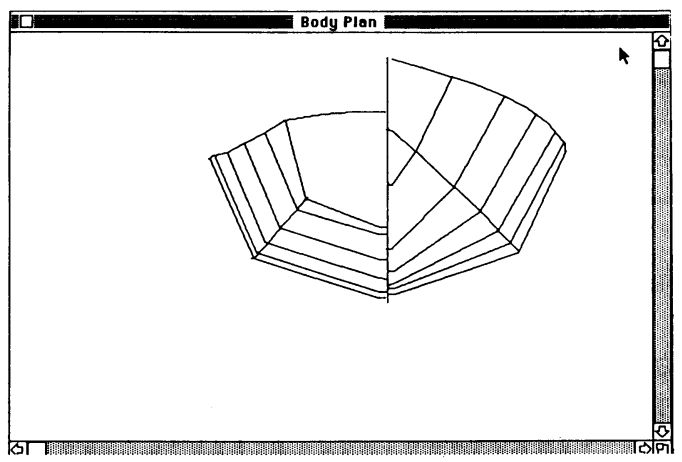

Fig. 17. Body plan of chined hull.

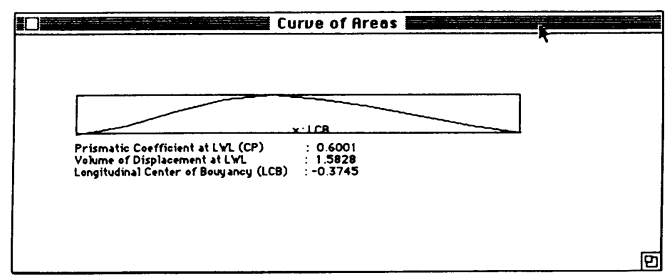

Fig. 18. Curve of areas plot of chined hull. able statistics, multi-variate statistics and to derived types of statistical analysis.

An example of using data to formulate preliminary descriptions is the triangular format of clusters of craft principal dimensions and other parameters taken from $\mathrm{FAO}^{9}$ (Fig. 20). It shows the linear relationship between the principal dimensions and the various
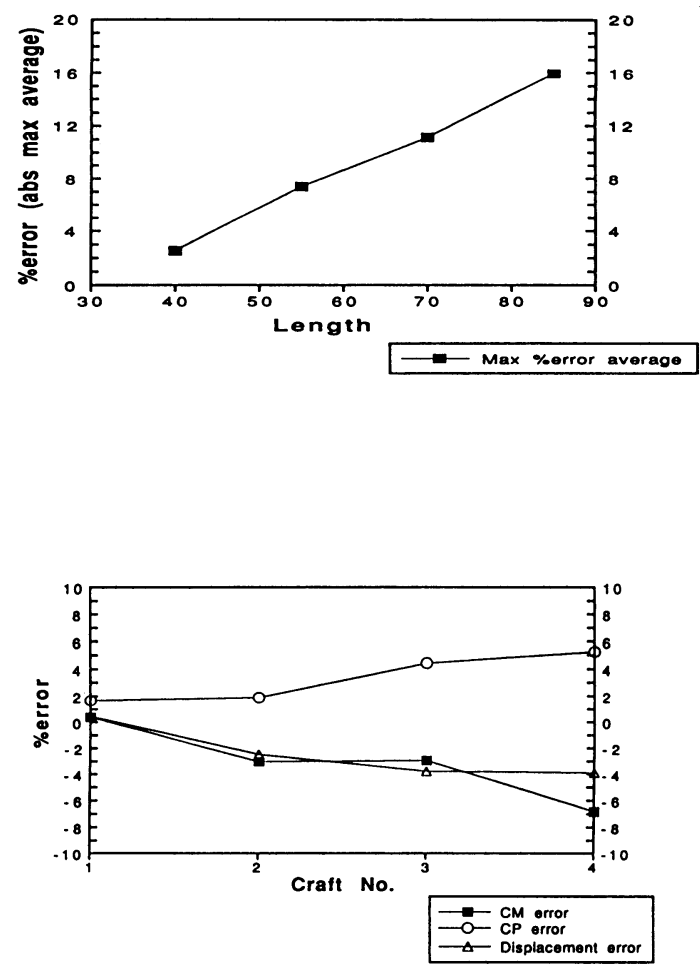

Fig. 19. Percentage error based on known parameters.

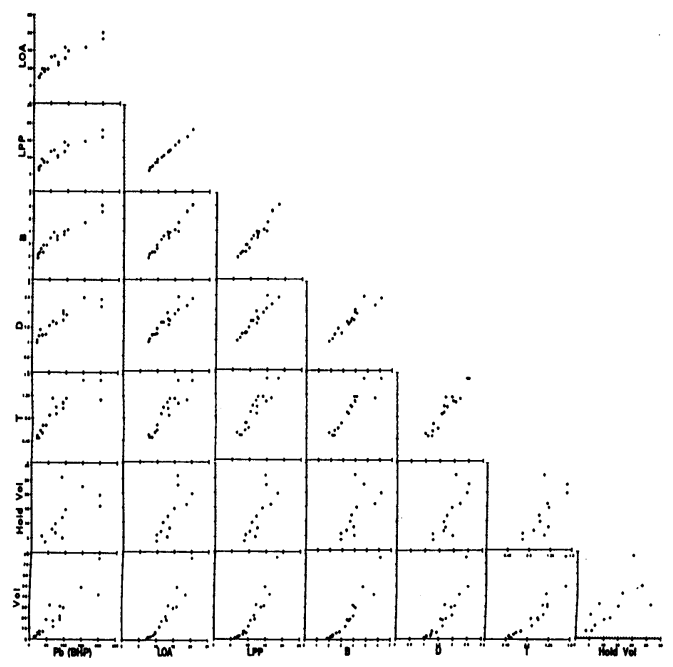

Fig. 20. Cluster layout for preliminary data view. 
strength of relationships between pairs also show some variance. Although such views of data are useful, they do not show dependencies or further information ${ }^{11}$.

Classification is a major task for statistics and also for the design problem. Several researches on fishing craft forms have been conducted and multi-variate techniques applied to classify hull forms. Before going into such sophisticated methods, a preliminary analysis of major groups would help in identifying the groupings themselves. Once such technique is the Andrews plot ${ }^{11}$ where the multiple variables are mapped into the form:

$$
\begin{aligned}
x(t)= & x_{1} / \sqrt{2}+x_{2} \sin (t)+x_{3} \cos (t) \\
& +x_{4} \sin (2 t)+x_{5} \cos (2 t) \cdots
\end{aligned}
$$

where :

$$
x_{1,2,3, \ldots, i}=i \text { variables } ;-\pi<t<\pi
$$

This classification technique was tried for a group of fishing crafts representing Japanese, FAO designed, Argentina and French trawlers. The plot (Fig. 21) clearly showed separate groupings. The mojor drawback to this technique is confusion when a number of objects are plotted; however, it does give a measure of classification for major groups.

Further work is intended employing multivariate analysis, probability theory and other appropriate statistical techniques to derive knowledge from existing data. The hulls represented in the data base will be used together with other available data in formulating empirical relationships, quantitative/qualitative rules and other proper forms comprising the design system.

\section{Conclusions}

An effort to develop programs for the acquisition of knowledge for the design of fishing crafts has been implemented and work is going on to further enhance the capabilities of the system. Three components for knowledge acquisitition are intended to be developed :

1) A consultation system for the creation of a knowledge base consisting of rules on decision making or

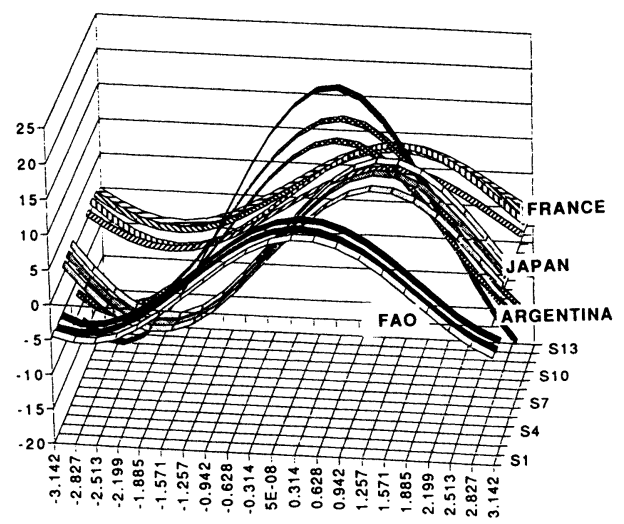

Fig. 21. Andrews plot of four craft groups. selection type problems that occur during the various phases of the design spiral.

2) A geometric input system developed in an object oriented system for the establishment of a database of fishing craft hulls.

3) Statistical techniques for derivation of knowledge from the database.

The geometry input system is currently employed to build up the data base of hull forms using published data from different sources. Using statistical techniques for analysis, the knowledge from such a database is to be derived and eventually employed in a knowledge based design system.

\section{References}

1) Rumblaugh, J. et. al. (1991). Object Oriented Analysis and Design. New York: Prentice-Hall International.

2) Neubert, S. and R. Studer (1992) "The KEEP Model, a Knowledge Engineering Process Model". Current Developments in Knowledge Acquisition-EKAW' '92, 6th European Knowledge Acquisition Workshop, Heidelberg and Kaiserslautern (ed) Wetter et al, Germany, May 18-22. Berlin : Springer-Verlag.

3) Boose, J. H. (1989) "A survey of Knowledge Acquisition Techniques and Tools", Knowledge Acquistition: An International Journal, Vol. 1, No. 1, pp. 3-38.

4) Boose, J. H. and J. M. Bradshaw (1987) "Expertise transfer and complex problems : using AQUINAS as a knowledge acquisition workbench for knowledge based systems". International Journal of Man-Machine Studies 26: ( 1 ) pp. 3-28. January.

5) Boose, J. H. (1986) Expertise transfer for expert system design. Amsterdam: Elsevier.

6) Stearns, H.P. Payne and G. Smith (1991). "Designing a Knowledge Based Ship Design System". Proceedings of the 4th International Marine Systems Design Conference. May Kobe Japan. p. 211.

7) Yamaguchi, F. (1978). "A New Curve Fitting Method using a CAT Computer Display”. Computer Graphics and Image Processing, p. 425-437.

8) FAO (1960). Fishing Boats of the World: 2. ed by J. Olof Traung, London: Fishing News Books.

9) FAO (1967). Fishing Boats of the World: 3. ed by J. Olof Traung. London: Fishing News Books.

10) Frawley, W. G. Piatetsky-Shapiro and C. Methues. (1992) "Knowledge Discovery in Databases: An Overview". Knowledge Discovery in Databases ed. by G. Piatetsky-Shapiro and W. Frawely, Menlo Park: AAAI Press/The MIT Press.

11) Everitt, B. and G. Dunn. (1992) Applied Multivariate Data Analysis. London : E. Arnold. pp. 30-35. 